\title{
Development of nanoemulsion for efficient brain parenteral delivery of cefuroxime: designs, characterizations, and pharmacokinetics
}

This article was published in the following Dove Press journal: International Journal of Nanomedicine

\author{
Siti Norhawani Harun' \\ Syafinaz Amin Nordin ${ }^{2}$ \\ Siti Salwa Abd Gani ${ }^{3,4}$ \\ Ahmad Fuad Shamsuddin ${ }^{5,6}$ \\ Mahiran Basri ${ }^{7}$ \\ Hamidon Bin Basri' \\ 'Department of Medicine, Faculty \\ of Medicine and Health Sciences, \\ Universiti Putra Malaysia, Serdang, \\ Malaysia; ${ }^{2}$ Department of Medical \\ Microbiology and Parasitology, \\ Faculty of Medicine and Health \\ Sciences, Universiti Putra Malaysia, \\ Serdang, Malaysia; ${ }^{3}$ Department of \\ Agriculture Technology, Faculty of \\ Agriculture, Universiti Putra Malaysia, \\ Serdang, Malaysia; ${ }^{4} \mathrm{Halal}$ Products \\ Research Institute, Universiti Putra \\ Malaysia, Serdang, Malaysia; ${ }^{5}$ Centre \\ for Drug Delivery Research, Faculty \\ of Pharmacy, Universiti Kebangsaan \\ Malaysia, Kuala Lumpur, Malaysia; \\ ${ }^{6}$ Faculty of Pharmacy and Health \\ Sciences, Universiti Kuala Lumpur \\ Royal College of Medicine Perak, Ipoh, \\ Malaysia; 'Department of Chemistry, \\ Faculty of Science, Universiti Putra \\ Malaysia, Serdang, Malaysia
}

Background and aim: Drugs that are effective against diseases in the central nervous system and reach the brain via blood must pass through the blood-brain barrier (BBB), a unique interface that protects against potential harmful molecules. This presents a major challenge in neuro-drug delivery. This study attempts to fabricate the cefuroxime-loaded nanoemulsion (CLN) to increase drug penetration into the brain when parenterally administered.

Methods: The nanoemulsions were formulated using a high-pressure homogenization technique and were characterized for their physicochemical properties.

Results: The characterizations revealed a particle size of $100.32 \pm 0.75 \mathrm{~nm}$, polydispersity index of $0.18 \pm 0.01$, zeta potential of $-46.9 \pm 1.39 \mathrm{mV}$, viscosity of $1.24 \pm 0.34 \mathrm{cps}$, and osmolality of $285.33 \pm 0.58 \mathrm{mOsm} / \mathrm{kg}$, indicating that the nanoemulsion has compatibility for parenteral application. CLN was physicochemically stable within 6 months of storage at $4{ }^{\circ} \mathrm{C}$, and the transmission electron microscopy revealed that the CLN droplets were almost spherical in shape. The in vitro release of CLN profile followed a sustained release pattern. The pharmacokinetic profile of CLN showed a significantly higher $C_{\max }$, area under the curve (AUC) $)_{0-t}$, prolonged half-life, and lower total plasma clearance, indicating that the systemic concentration of cefuroxime was higher in CLN-treated rats as compared to cefuroxime-free treated rats. A similar profile was obtained for the biodistribution of cefuroxime in the brain, in which CLN showed a significantly higher $C_{\max }$, $\mathrm{AUC}_{0-t}$, prolonged half-life, and lower clearance as compared to free cefuroxime solution.

Conclusion: Overall, CLN showed excellent physicochemical properties, fulfilled the requirements for parenteral administration, and presented improved in vivo pharmacokinetic profile, which reflected its practical approach to enhance cefuroxime delivery to the brain.

Keywords: parenteral nanoemulsion, cefuroxime, drug delivery, pharmacokinetics, bloodbrain barrier

\section{Introduction}

Diseases of the central nervous system (CNS) such as meningitis, schizophrenia, migraine, Parkinson's disease, and Alzheimer's disease require delivery of the drug to the brain for treatment. Meningitis (infection of the meningeal layer) is one of the commonest and most debilitating acute neurological conditions. ${ }^{1,2}$ High-dose antibiotics were used to treat CNS diseases with a significant increase in side effects. ${ }^{3,4}$ Drugs that are effective against diseases in the CNS and reach the brain via the blood compartment must pass through the blood-brain barrier (BBB). Many drugs are not effective in the CNS because of their limited lipid solubility for BBB penetration. The entry of drugs, including antibiotics, into the cerebrospinal fluid (CSF) and extracellular space of the brain is governed by molecular size, lipophilicity, and plasma-binding protein. ${ }^{5}$ The present studies propose the utilization of nanoemulsion
Correspondence: Hamidon Bin Basri Department of Medicine, Faculty of Medicine and Health Sciences, Universiti Putra Malaysia, 43400 Serdang, Selangor, Malaysia

Tel +60389472563

Fax +60389472759

Email hamidon@upm.edu.my 
as a lipid nanosystem efficient that improves antibiotic (cefuroxime) delivery to the brain for the treatment of meningitis.

Cefuroxime is a broad-spectrum second-generation cephalosporin antibiotic that is active against $\beta$-lactamase-producing strains. It is a semisynthetic analog of cephalosporin-C and the first of a series of $\alpha$-metoxyiminoacyl-substituted cephalosporins. ${ }^{6}$ The chemical structure of cefuroxime is shown in Figure 1. Cefuroxime may reach the CSF through diffusion or active transport. ${ }^{7}$ However, further studies are recommended in regard to transport mechanisms of cefuroxime across $\mathrm{BBB}$. The recent finding showed that for cephalosporins, a significant relationship was found between lipophilicity and diffusion across the BBB in rats. ${ }^{8}$ In the presence of brain inflammation, cefuroxime has superior penetration into the CSF compared to other cephalosporins and showed relatively low toxicity. ${ }^{7}$ The incorporation of cefuroxime in oil core of nanoemulsion increases the permeability of the drug via passive diffusion by manipulating the lipid solubility to increase drug delivery to the brain.

Among the attractive features offered by lipid emulsions' delivery systems are stability and safety records. Parenteral route is one of the most common and effective routes for drug administration that is usually adopted for active molecules with low bioavailability and narrow therapeutic index. Lipid emulsion dissolves hydrophobic drugs and protects them from hydrolysis and enzymatic degradation, criteria that make nanoemulsions ideal vehicles for parenteral transport. ${ }^{9}$ Furthermore, the side effects of administered drugs will decrease through the reduction of the frequency and dosage of injections throughout the drug therapy period as these emulsions guarantee the release of drugs in a sustained and controlled mode over long periods. Additionally, the large interfacial area in nanoemulsions enhances drug transport and delivery, as well as targeting them to specific sites. ${ }^{10}$

To investigate the efficiency of cefuroxime delivery to the brain, the effects of the type of oil and surfactant on cefuroxime solubility were studied. Cefuroxime-loaded nanoemulsion (CLN) was also evaluated for entrapment efficiency (EE), physical stability, in vitro drug release, and

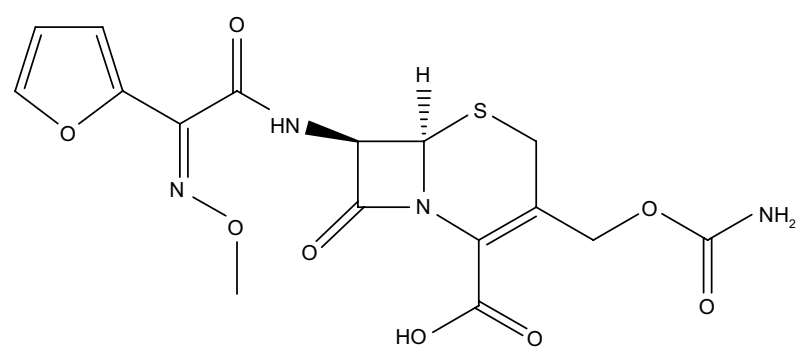

Figure I The molecular structure of cefuroxime. cytotoxicity study. The therapeutic availability was studied through comparison of CLN pharmacokinetic profile with free drug solution.

\section{Materials and methods Materials}

Cefuroxime, $(6 R, 7 R)-3-\{[($ aminocarbonyl)oxy $]$ methyl $\}-7-$ $\{[(2 Z)-2$-(2-furyl)-2-(methoxyimino)acetyl $]$ amino $\}-8$-oxo5-thia-1-azabicyclo[4.2.0]oct-2-ene-2-carboxylic acid, was purchased from Euroasias Chemical Private Limited (Mumbai, India). Safflower seed oil, soybean oil, sunflower oil, pine nut oil, olive oil, Cremophor EL, and sodium oleate were purchased from Sigma-Aldrich Co. (St Louis, MO, USA). Lecithin Lipoid S75 was also purchased from Lipoid GmbH (Ludwigshafen, Germany); Tween 80 (polyoxyethylene sorbitan monooleate) was purchased from Merck (Hohenbrunn, Germany), and glycerol was purchased from J.T. Baker (Phillipsburg, NJ, USA). All the used oils and surfactants were analytically graded materials. Acetonitrile (highperformance liquid chromatographic [HPLC] grade, 99.9\% purity) was purchased from J.T. Baker. Sodium hydroxide (analytical grade) was purchased from Merck Millipore (Billerica, MA, USA). Water was deionized and Milli-Q filtered. Centrisart tubes and dialysis membranes were from Sartorius AG (Göttingen, Germany). All other chemicals and reagents were of analytical or HPLC grade.

\section{HPLC analysis}

Waters Acquity High Performance Liquid Chromatographic system (Waters Corporation, Milford, MA, USA), which was equipped with a photodiode array (PDA) detector (Waters 2998) and auto samplers, was used for cefuroxime analysis. The HPLC used the Empower 3 Chromatography software. The column used was Chromolith ${ }^{\circledR}$ HighResolution RP-18 Endcapped $100(4.6 \mathrm{~mm})$ with guard cartridge kit $(4.6 \mathrm{~mm})$. The mobile phase consisted of a mixture of deionized water and acetonitrile (40:60 ratio). The detection wavelength was $275 \mathrm{~nm}$ under an isocratic flow of $1.0 \mathrm{~mL} / \mathrm{min}$. All analyses were performed at $25^{\circ} \mathrm{C}$ and $20 \mu \mathrm{L}$ injection volume for the sample injection used. This method was previously validated according to International Council for Harmonisation of Technical Requirements for Pharmaceuticals for Human Use (ICH) Guidelines and proven specific, linear $\left(r^{2}>0.9995\right)$, and precise (relative standard deviation $[\mathrm{RSD}]<1.85 \%$ ).

\section{Solubility studies of cefuroxime in different oils}

The solubility assay of cefuroxime in different types of oil was performed to determine the oil that can highly solubilize 
cefuroxime. The tested oils include corn oil, castor oil, palm oil, palm kernel oil ester, soybean oil, middle-chain triglyceride (MCT), sunflower oil, safflower seed oil, safflower oil, flaxseed oil, sesame oil, and pine nut oil. An excess amount of cefuroxime was gradually added to the selected oils at a ratio of 1:1 with the presence of lecithin. The solution was kept under moderate magnetic stirring (IKA, Shanghai, China) for $24 \mathrm{~h}$ to reach equilibrium. The sample was then centrifuged (Hettich Zentrifugen, Tuttlingen, Germany) at 4,000 rpm for $15 \mathrm{~min}$. An aliquot of the supernatant was diluted with acetonitrile. The cefuroxime content was analyzed by HPLC.

\section{Preparation of CLN}

CLN was formulated using high-pressure homogenization method. The oil phase was prepared by adding cefuroxime $(0.3 \%, \mathrm{w} / \mathrm{w})$ into the oil mixture $(10 \%, \mathrm{w} / \mathrm{w})$ and lecithin $(3 \%$, $\mathrm{w} / \mathrm{w})$ as a surfactant. Then, Tween $80(0.8 \%$, w/w $)$ was added after cefuroxime was completely dissolved. The oil phase was added dropwise into the aqueous phase that contained glycerol $(2.3 \%, \mathrm{w} / \mathrm{w})$ and sodium oleate $(0.1 \%, \mathrm{w} / \mathrm{w})$. This solution was continuously stirred using an overhead stirrer (RW 20 digital; IKA) for $\sim 1 \mathrm{~h}$. Through this method, the coarse emulsion was formulated. The prepared coarse emulsions were then subjected to higher energy emulsification techniques to produce very fine, nanosized CLN. A highpressure homogenizer was used for six cycles at 1,000 bar (EmulsiFlex; Avistin, Inc., Ottawa, ON, Canada). A blank formulation was also prepared. The formulation composition is presented in Table 1.

\section{Characterizations of CLN}

$\mathrm{CLN}$ was kept at $4^{\circ} \mathrm{C}$ and characterized with respect to particle size measurement, polydispersity index (PDI), zeta potential (ZP), osmolality, viscosity, microscopic study, toxicity study, in vitro release profile, and drug encapsulation efficiency.

Table I The constituents of CLN prepared by high-pressure homogenizer

\begin{tabular}{ll}
\hline Composition & Amount (\%, w/w) \\
\hline Cefuroxime & 0.30 \\
Lecithin & 3.00 \\
Tween 80 & 0.80 \\
Glycerol & 2.30 \\
Q-tocopherol & 1.00 \\
Sodium oleate & 0.10 \\
Soybean oil & 5.00 \\
Sunflower seed oil & 5.00 \\
Deionized water & 82.50 \\
Total & 100.00 \\
\hline
\end{tabular}

Abbreviation: CLN, cefuroxime-loaded nanoemulsion.

\section{Particle size, PDI, and ZP measurements}

Particle size and PDI were measured using Malvern Nano ZS90 (Malvern Instruments, Malvern, UK) that was operated based on dynamic light scattering (DLS) at $25^{\circ} \mathrm{C}$. A total of $1 \mathrm{~mL}$ of CLN sample was diluted with deionized water until it reached the desired concentration. The count rate for all diluted samples was in the range of 150-300 kcps. These parameters were evaluated in conjunction with the stability study for a period of 6 months at both $25^{\circ} \mathrm{C}$ and $4^{\circ} \mathrm{C}$. All samples were measured in triplicate.

\section{Osmolality measurement}

The osmolality value of CLN was recorded using an osmometer, based on the principle of freezing point method (Model 3320; Advance Instruments, Inc., Norwood, MA, USA). The osmometer was calibrated with a reference standard of 100 and 3,000 mOsm/kg (Advance Instruments, Inc.). A total of $0.25 \mathrm{~mL}$ sample was analyzed, and the measurement was repeated thrice.

\section{Viscosity evaluation}

The viscosity was analyzed using a viscometer with SC4-18 spindle (Brookfield DV-II+; Brookfiled, Middleboro, MA, USA). The spindle was completely immersed into the sample at a maintained temperature of $25^{\circ} \mathrm{C}$. Once the spindle started to rotate, the viscous sample will move against the spindle wall. Deflection of the calibrated spring was measured as the viscosity of tested sample.

\section{Microscopic study of nanoemulsion}

Conventional negative staining using $1 \%(\mathrm{w} / \mathrm{v})$ uranyl acetate was used for microscopic visualization of nanoemulsion using transmission electron microscope (TEM) (LEO 912AB; LEO, Peabody, MA, USA). A droplet of CLN was placed on a Formvar carbon-coated copper grid, 300 mesh (Electron Microscopy Sciences, Hatfield, PA, USA), and stained with $50 \mu \mathrm{L}$ uranyl acetate. The stained sample was allowed to dry at room temperature for $10 \mathrm{~min}$. Excess liquid was removed using filter paper, and the grid was observed through TEM.

\section{Cytotoxicity study}

Ten thousand $(10,000)$ cells/well of human brain endothelial cells, hCMEC/D3, were seeded into 96-well plates. The hCMEC/D3 cells were obtained from Dr Pierre-Olivier Couraud of the Institut Cochin, INSERM, Paris, and first described by Weksler et al (2005). ${ }^{11}$ The cells were incubated for $24 \mathrm{~h}$ to allow cell attachment. A stock solution of $1,000 \mu \mathrm{g} / \mathrm{mL}$ concentration was prepared for a standard cefuroxime sample, and nanoemulsion was loaded with cefuroxime. A log series 
of $100,10,1$, and $0.1 \mu \mathrm{g} / \mathrm{mL}$ concentrations were prepared by a serial dilution method. A total of $20 \mu \mathrm{L}$ of each sample concentration was added to each well to yield a final concentration. The prepared plate was then incubated at $5 \% \mathrm{CO}_{2}$ and $37^{\circ} \mathrm{C}$ for $48 \mathrm{~h}$. Next, $50 \mu \mathrm{L}$ of 3-(4,5-dimethythiazol-2-yl)-2, 5-diphenyl tetrazolium bromide (MTT) reagent was added to each well and incubated for another $4 \mathrm{~h}$ to form an insoluble, dark purple-colored formazan product. The cell media were carefully aspirated from the well without disturbing the purple precipitate and cell attached on the plate surface. A total of $100 \mu \mathrm{L} /$ well of dimethyl sulfoxide (DMSO) was then added to each well to dissolve the formazan product. The number of viable cells was measured using the SOFTProMax spectrometer at an absorbance wavelength of $550 \mathrm{~nm}$.

\section{In vitro release study of CLN}

The permeation study of CLN was carried out using modified Franz diffusion cells (PermeGear, Riegelsville, PA, USA). ${ }^{12}$ Cellulose acetate membrane with a molecular weight cutoff of 12,000 Da and an average pore size of 2.4 nm was placed between two compartments of Franz cell and was tightly clipped. This provided a $4.9 \mathrm{~cm}^{2}$ diffusion area between medium. A total of $1 \mathrm{~mL}$ of nanoemulsion containing cefuroxime and buffer mixture (1:2) was placed into the donor medium, on top of the membrane, and a $20 \mathrm{~mL}$ mixture of phosphate-buffered solution (PBS; $\mathrm{pH} 7.4$ ) and acetonitrile (4:1) was filled inside the receptor medium below the membrane. Acetonitrile was added to the receptor medium to increase cefuroxime solubility in the aqueous buffer. The temperature was maintained at $37^{\circ} \mathrm{C}$, and the stirring rate was set at $600 \mathrm{rpm}$. Sampling point was set up at various intervals $(1,2,3,4,6,8,10,12,24$, and $36 \mathrm{~h})$. For sampling, $1 \mathrm{~mL}$ of PBS solution was taken from the receptor medium, and another $1 \mathrm{~mL}$ of the fresh PBS was immediately replaced with the same receptor medium. The quantitative analysis of cefuroxime in $1 \mathrm{~mL}$ of the sample, which was taken from the receptor medium of Franz cells, was assayed using the HPLC method, as described in the "HPLC analysis" section. Cumulative percentage of drug release vs time was plotted to evaluate the drug release pattern from a drug solution and the CLN.

\section{Encapsulation efficiency study}

Ultrafiltration method using the Centrisart tube (molecular weight cutoff of 10,000 Da; Sartorius AG, Göttingen, Germany) was used to determine the encapsulation efficiency of CLN. ${ }^{13}$ Next, $2.0 \mathrm{~mL}$ of sample was placed in the outer chamber and centrifuged at 3,500 rpm for $15 \mathrm{~min}$.
The aqueous phase was collected from the floater that was inserted into the tube after centrifugation. The resultant aqueous phase was analyzed using HPLC conditions, as previously mentioned in the "HPLC analysis" section, to estimate the amount of drug (cefuroxime). Cefuroxime concentration entrapped in the emulsion system was calculated from the difference between the total and the free drug concentrations in the ultrafiltrate.

\section{Stability evaluation of nanoemulsion}

CLN storage stability was investigated by measuring the particle size, PDI, and ZP, as described in the "Particle size, PDI, and ZP measurements" section. CLN drug content was analyzed in respect to their drug-loading periods at 0,3 , and 6 months.

\section{In vivo studies}

Pharmacokinetic profile of CLN was evaluated through an in vivo CLN study. All experiments were performed in accordance with the guidelines for the care and use of laboratory animals upon getting approval from the Institutional Animal Care and Use Committee (IACUC), Universiti Putra Malaysia.

\section{Animal model}

Male Sprague Dawley rats (weighing 250-300 g) were used for animal study and housed in the animal house of Comparative Medicine and Technology Unit (COMeT), Universiti Putra Malaysia. The animals were placed in collective cages ( size $43 \times 27 \times 15 \mathrm{~cm}$ ), with not more than four animals per cage with poplar shaving bedding under controlled conditions $22^{\circ} \mathrm{C} \pm 2{ }^{\circ} \mathrm{C}, 55 \% \pm 10 \%$ relative humidity, and a $12 \mathrm{~h}$ light-dark cycle. They were supplied with a standard diet ad libitum and have free access to tap water ad libitum.

\section{In vivo experimental design}

The rats fasted overnight with free access to water ad libitum before the experimental day. On the experimental day, rats from the group of interest were firmly restrained. A total of $20 \mathrm{mg} / \mathrm{kg}$ dose of cefuroxime-free drug solution was dissolved in normal saline/CLN and intraperitoneally injected on each rat. CLN was sterilized by filtration through a $0.22 \mu \mathrm{m}$ membrane filter prior to injection. The animals were kept unrestrained during the entire study. At 15, 30, 60,90 , and $120 \mathrm{~min}$, five animals were anesthetized by ketamine $(100 \mathrm{mg} / \mathrm{mL})$ :xylazine $(20 \mathrm{mg} / \mathrm{mL})$ via intraperitoneal route. After the animals were completely anesthetized, 
blood sample was withdrawn by cardiac puncture into the preheparinized polypropylene tube. Then, the animals were immediately decapitated and the brain samples were collected. The blood samples were centrifuged at 3,500 rpm for $10 \mathrm{~min}$ to isolate plasma sample. The brain tissues were weighed, washed, and homogenized with PBS (pH 7.4). Perchloric acid (70\%) (1:10) was added to the brain tissues and vortexed for $5 \mathrm{~min}$. The resulting solution was then centrifuged at 13,000 rpm for $5 \mathrm{~min}$, and the supernatant was collected. The collected plasma and brain homogenates were stored at $-80^{\circ} \mathrm{C}$ until analysis.

\section{Pharmacokinetic study of CLN}

The untreated plasma samples and brain homogenate were used to prepare $0.5,1.0,5.0,10.0,15.0,30.0$, and $50.0 \mu \mathrm{g} / \mathrm{mL}$ standard concentrations of cefuroxime. Cooled acetonitrile was used as the deproteinization agent before being introduced to HPLC analysis. Then $400 \mu \mathrm{L}$ of ice-cold acetonitrile was added to $200 \mu \mathrm{L}$ of plasma samples or brain homogenates. The resulting mixture was vortexed for $5 \mathrm{~min}$ and centrifuged at 13,000 rpm for $5 \mathrm{~min}$. The supernatant evaporated to dry under a stream of nitrogen, and the residue was reconstituted in $200 \mu \mathrm{L}$ of $10 \%$ acetonitrile aqueous solution $(\mathrm{v} / \mathrm{v})$. The resulting solution was transferred to an autosampler vial, and $20 \mu \mathrm{L}$ of aliquot was injected into the HPLC system. A standard calibration curve was obtained by plotting the peak area ratios extracted from HPLC against the concentration of cefuroxime. This calibration curve was manipulated to quantitate the concentration of cefuroxime in the treated plasma and brain tissue homogenates.

\section{Statistical analysis}

All values were demonstrated as mean \pm SD from at least three independent experiments. The statistical differences between the groups were tested by using Student's $t$-test, and with more than two groups, analysis of variance (ANOVA) was used for the comparison of results. The difference was considered statistically significant if $P<0.05$.

\section{Results and discussion Determination of cefuroxime solubility in oils}

Determination of oil core was the first step prior to nanoemulsion fabrication, and the high solubility of drug usually drives the oil selection. ${ }^{14}$ The solubility of the drug in oil was crucial to achieve optimum drug loading and to prevent the drug precipitation after emulsification. The solubility of cefuroxime in different oils was assessed using the HPLC method, as described in the "HPLC analysis" section, and the solubility study is presented in Figure 2.

Among the tested oils, cefuroxime presented the highest solubility in the oil mixture of soybean oil and sunflower oil $(50 / 50, \mathrm{w} / \mathrm{w})$. Soybean oil was also found as the reliable source of essential fatty acid in the form of linoleic acid (w-6 FA) and $\alpha$-linolenic acid (w-3 FA). It was hypothesized that a higher content of linoleic acid can enhance drug penetration through the BBB by simple diffusion. ${ }^{15}$ The previous study also suggested simple diffusion as the primary route of fatty acid passage across the $\mathrm{BBB}$, and a recent study had acknowledged the potential involvement of fatty acid transport proteins. ${ }^{16}$

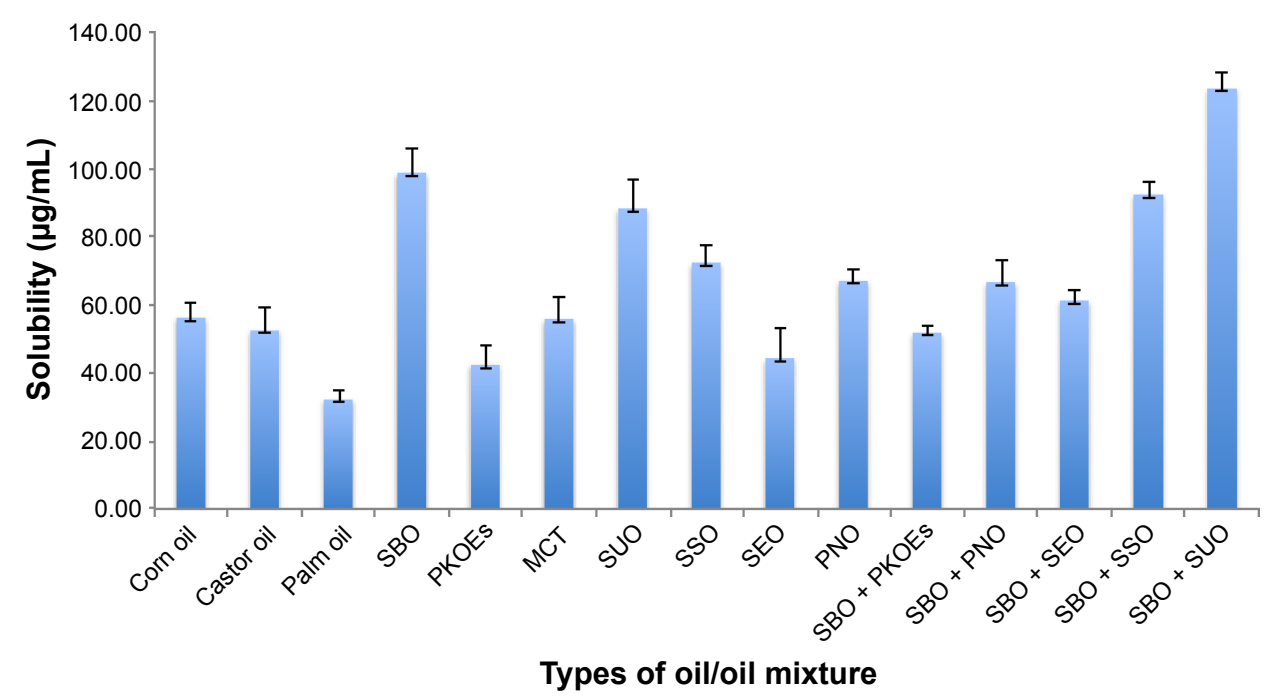

Figure 2 Solubility of cefuroxime in different types of oils and oil mixtures.

Note: Error bars denote standard deviation $(n=3)$.

Abbreviations: MCT, middle-chain triglyceride; PKOEs, palm kernel oil esters; PNO, pine nut oil; SEO, sesame oil; SBO, soybean oil; SSO, safflower seed oil; SUO, sunflower oil. 


\section{Fabrication of CLN}

In practice, the usage of oil often requires the addition of other additives/surfactants to stabilize the nanoemulsion system. The presence of surfactant created the interfacial tension gradients that formed the energy barrier against droplet breakage in an emulsion system. ${ }^{17}$ Lecithin was chosen as the main surfactant due to its safety reputation, and they are currently used in commercial lipid emulsions. ${ }^{18-20}$ Furthermore, lecithin as a naturally occurring phospholipid derived from soybean contains a phospholipid and acetylcholine that was demonstrated to have a profound effect on brain function. The percentage of lecithin used in nanoemulsion productions was kept in a permitted concentration of $3.00 \%(\mathrm{w} / \mathrm{w}){ }^{21} \mathrm{Few}$ co-surfactants were screened to assist the cefuroxime-loading capability in oil phase as well as to improve the CLN stability (Figure 3).

As demonstrated in Figure 3, cefuroxime was mostly dissolved in Tween $80(95.77 \pm 3.11 \mu \mathrm{g} / \mathrm{mL})$ followed by lecithin $(88.73 \pm 3.23 \mu \mathrm{g} / \mathrm{mL})$, chromophore EL $(76.54 \pm 2.35 \mu \mathrm{g} / \mathrm{mL})$, and glycerol $(75.65 \pm 2.33 \mu \mathrm{g} / \mathrm{mL})$. Lecithin, as the main surfactant, conferred good solubility behavior and was proven as a suitable carrier for lipophilic drug. Numerous studies on nanoparticle usage as carriers coated with polysorbate, such as Tween 80 , were reported to have successfully passed through the BBB. ${ }^{22,23}$ For nanoemulsion, Tween 80 had increased the nanoemulsion-loaded drug uptake by adsorption-mediated endocytosis via the low density lipoprotein (LDL) receptor of brain endothelial cells. Nanoemulsion then mimicked the lipoprotein, and the drug was released within the endothelial cells by passive diffusion into the brain or transported into the brain by transcytosis. Additionally, Tween 80 has the ability to inhibit P-glycoprotein that causes drug efflux in the CNS. ${ }^{13}$ Considering all the above advantages, lecithin and Tween 80 were selected as the surfactant mixture for the fabrication of CLN.

D- $\alpha$-tocopherol, commonly known as vitamin E, was incorporated into the nanoemulsion system due to its

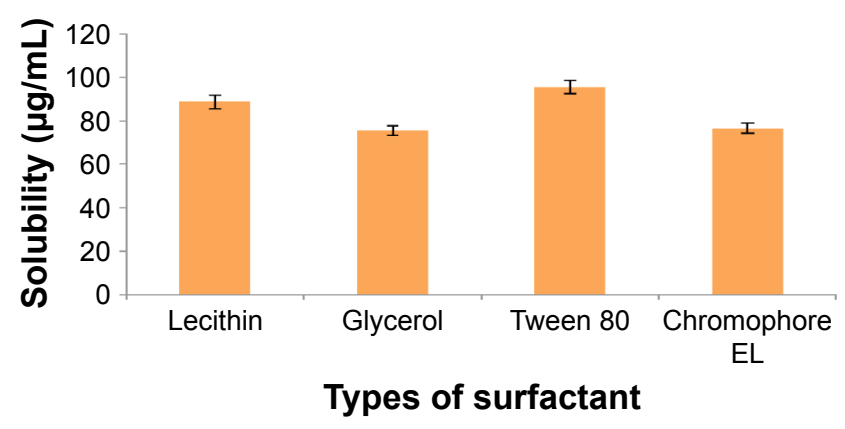

Figure 3 Solubility of cefuroxime in different types of surfactant solution. Note: Error bars denote standard deviation $(n=3)$. antioxidant effect. ${ }^{24}$ Generally, soybean oil possessed its own antioxidant effect when used as an oil phase in an emulsion system. However, due to the high concentration of poly-unsaturated fatty acids (PUFAs), the use of soybean oil in the emulsion may reduce $\alpha$-tocopherol in plasma lipoproteins and deplete antioxidant defenses. ${ }^{25}$ Hence, to prevent the oxidation of cefuroxime in the oil phase, $1 \%$ of $\alpha$-tocopherol was added into the CLN formulation. To maintain the nanoemulsion system isotonicity, the osmolality value was kept between 280 and $310 \mathrm{mOsm} / \mathrm{kg}$, the osmolality limits for parenteral preparation. Osmolality value of CLN was controlled by the addition of glycerol (2.3\%). A small amount of sodium oleate $(0.1 \%)$ was incorporated into the CLN system to maintain the ZP value within the acceptable limit. The addition of small amount of sodium oleate was found to markedly increase the ZP value in the lipid delivery of vinorelbine and enhance its stability as demonstrated by Zhang et al. ${ }^{26}$

\section{Characterizations of CLN}

\section{Particle size, ZP, and PDI analyses}

These three parameters were evaluated using Malvern Zetasizer, a dynamic laser light scattering particle size analyzer. This instrument also incorporates a ZP analyzer that uses electrophoretic light scattering for particles, molecules, and surfaces, and a molecular weight analyzer using static light scattering. Fresh CLN has a particle of size $100.32 \pm 0.75 \mathrm{~nm}$ and a minor change of particle size that occurred within 6 months of stability assessment period $\left(4^{\circ} \mathrm{C}\right.$ of storage). $\mathrm{PDI}$ and $\mathrm{ZP}$ values were within the parenteral limit (data are presented in the "Stability evaluation of CLN" section). The minute size of oil droplet was crucial for long-term stability as the small droplet resisted physical destabilization caused by gravitational separation, flocculation, and/or coalescence. ${ }^{27}$ The diameter of blood capillaries was $\sim 5-10 \mu \mathrm{m}$; thus, the carrier size should be smaller than this size range in order to be circulated in the blood vessel. ${ }^{28}$ Hence, the particle size of CLN was feasible for systemic delivery in blood.

The PDI reflects the uniformity and the distribution of oil droplet. A small PDI of $<0.2$ indicates a narrow and concentrated particle size distribution and thus better stability against destabilization. ${ }^{29,30}$ For parenteral application, PDI value up to 0.250 was acceptable. ${ }^{31}$ In the present study, the particle size analysis (Table 2) confirmed that the particle diameter of CLN was within the submicron range with the average reading of $\sim 100 \mathrm{~nm}$. There was a minimal increase in particle size observed after 3 months, but the changes were under the acceptable limit for stability assessment. During the 
Table 2 Physicochemical properties of freshly prepared CLN

\begin{tabular}{ll}
\hline Parameter & Value \\
\hline Particle size $(\mathrm{nm})$ & $100.32 \pm 0.75$ \\
Polydispersity index & $0.18 \pm 0.0 \mathrm{I}$ \\
Zeta potential $(\mathrm{mV})$ & $-46.90 \pm 1.39$ \\
Viscosity $(\mathrm{mPa} \mathrm{s})$ & $1.24 \pm 0.34$ \\
Osmolality $(\mathrm{mOsm} / \mathrm{kg})$ & $285.33 \pm 0.58$ \\
\hline
\end{tabular}

Note: All measurements were performed in triplicate.

Abbreviation: CLN, cefuroxime-loaded nanoemulsion.

assessment period, the PDI value varied from 0.17 to 0.25 , indicating a relatively narrow particle size distribution and adequate for the parenteral application. Judging from the variation of PDI value, the particle size of CLN may exist as a polydisperse suspension but still within the parenterally acceptable limit. Samples were considered polydisperse when the PDI was $>0.2 .^{27}$

$\mathrm{ZP}$ analysis was directly related to the electrophoretic mobility of the particles, and the value was calculated from the measured velocity. ${ }^{32}$ If the droplets are highly charged, then a strong electrostatic repulsion will be set up between them, which will prevent coalescence. If the ZP was reduced, the emulsion can become unstable as the electrostatic repulsion can no longer overcome the momentum of the colliding particles. ${ }^{33}$ $\mathrm{ZP}$ values $>+30$ or $<-30 \mathrm{mV}$ typically offered high degrees of stability. ${ }^{34} \mathrm{ZP}$ evaluation revealed that CLN exhibited average negative $\mathrm{ZP}$ values with a reading of $<-30 \mathrm{mV}$, indicating good electrochemical stability. The negative charge conferred by CLN may be due to fatty acid contents of the soybean oil and the interfacial phospholipid properties of lecithin. ${ }^{35}$ Negatively charged phospholipid increased the droplet repulsion and subsequently decreased flocculation, which ensured better emulsion stability. ${ }^{36}$

\section{Viscosity and osmolality analyses}

Viscosity measurement relates to flow characteristic of a system; thus, for the parenteral delivery product, the formulation should exhibit good syringeability (easily drawn into a syringe) and injectability (readily rejected from the syringe). ${ }^{37}$ A study on parenteral nanoemulsion conducted by Araújo et $\mathrm{al}^{14}$ reported that the acceptable value for parenteral administration was $<3.9 \mathrm{mPa}$ s. The viscosity value of CLN was found to be $1.24 \pm 0.34 \mathrm{mPa}$ s (Table 2), and this value was within the specification for parenteral emulsions. The low viscosity value presented by CLN formulation reflected the stability and safety measure prior to its application.

The parenteral product should be isotonic and euhydric (physiological $\mathrm{pH}$ ) to avoid local damage on the vascular endothelium and circulating blood cells. ${ }^{38}$ Incorporation of the small amount of glycerol assisted in generating emulsion with osmolality value that is close to the normal osmotic pressure at physiological $\mathrm{pH}(280-320 \mathrm{mOsm} / \mathrm{kg})$. The addition of $2.3 \%$ of glycerol $(\mathrm{w} / \mathrm{w})$ has significantly increased the osmolality value of CLN to $285.33 \pm 0.58 \mathrm{mOsm} / \mathrm{kg}$, the value that was isotonic to blood.

\section{Morphology of CLN}

Prior to TEM analysis, conventional negative staining using uranyl acetate was performed. Staining allows identification of dehydrated shells of the nanoemulsion droplets, which are stabilized by the surfactant. As demonstrated in the TEM micrographs (Figure 4), CLN oil droplet was spherical in shape (A) and quite well distributed (B), which was in agreement with the result obtained by the DLS method. By using the DLS method, the mean particle size for CLN was $\sim 100 \mathrm{~nm}$ and the PDI value ranged from 0.17 to 0.25 , suggesting the polydispersity (variation of size) of the oil droplet. The morphology of CLN oil droplets obtained in this study was in accordance with TEM photomicrographs of nanoemulsion presented in other nanodelivery studies. ${ }^{14,39,40}$

\section{Cytotoxicity of CLN}

In vitro cytotoxicity testing has become an integral aspect of drug discovery because it is a convenient, cost-effective, and predictive mean of characterizing the toxic potential of new chemical entities. ${ }^{41}$ For CLN quantitative data of toxicity test, MTT assay was employed. As demonstrated in Figure 5, cefuroxime standard did not show any toxic effect to the cells even if a high concentration was applied compared to blank nanoemulsion (nanoemulsion without cefuroxime) and CLN. The most likely explanation for this difference was
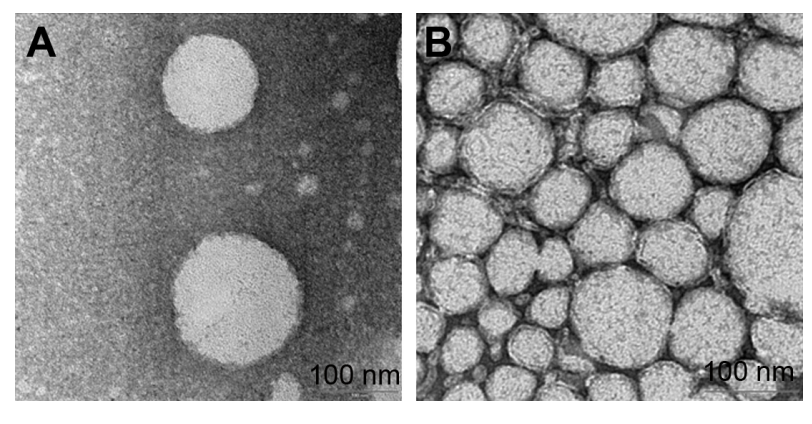

Figure 4 TEM photomicrographs of cefuroxime-loaded nanoemulsion after negative staining with uranyl acetate.

Notes: (A) The image of oil droplet after 100x dilution. (B) The image of oil droplet after $50 \times$ dilution. The scale bars represent $100 \mathrm{~nm}$.

Abbreviation: TEM, transmission electron microscope. 


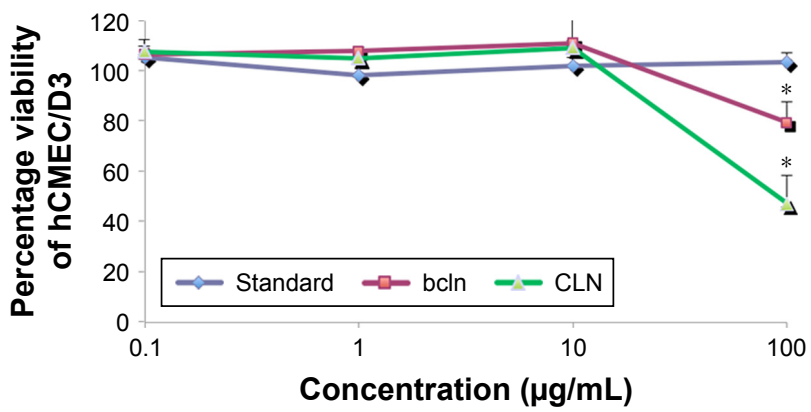

Figure 5 Dose-response curve of CLN.

Notes: The cytotoxicity effect of the formulation against hCMEC/D3 cell line as determined by MTT assay after $24 \mathrm{~h}$ exposure to cefuroxime standard solution, bcln, and CLN. Error bars indicate the standard deviation of three independent experiments. *Significant values $(P<0.05)$ in comparison with free cefuroxime solution.

Abbreviations: bcln, blank nanoemulsion; CLN, cefuroxime-loaded nanoemulsion; MTT, 3-(4,5-dimethythiazol-2-yl)-2,5-diphenyl tetrazolium bromide.

the incorporation of the surfactant and co-surfactant into the nanoemulsion system. The review of surfactant toxicity as related to the pharmaceutical application has been published in literatures. ${ }^{42,43}$

Few mechanisms should be considered in explaining how the surfactants can damage cell membranes, which resulted from the integrity loss of cell function. First, the surfactant at low concentration (below critical micelle concentration $[\mathrm{cmc}])$ may partition into the cell membrane and will cause the detrimental effect by altering the cell membrane integrity. Second, when the surfactant was added at concentrations above its cmc, it formed aggregates that will solubilize the cell membrane components and subsequently damage the cell membrane. ${ }^{43}$ Despite the surfactant toxic effect presented, careful selection of surfactant with optimum concentration was practiced to provide a balance between minimum toxic level and stable nanoemulsion system for drug delivery application. This toxicity test also revealed that CLN was nontoxic at the concentration of $10 \mu \mathrm{g} / \mathrm{mL}$. This concentration is acceptable for minimum inhibitory concentration (MIC) for cefuroxime. The therapeutic serum concentration of cefuroxime was $\sim 2 \mu \mathrm{g} / \mathrm{mL}$, hence the cytotoxicity assay proved that CLN is nontoxic up to $10 \mu \mathrm{g} / \mathrm{mL}$, reflecting its safety for therapeutic application.

\section{In vitro release profile of CLN}

In vitro release is an indirect measurement of drug availability, especially in preliminary stages of product development. In vitro release profile reveals fundamental information on the structure and behavior of the formulation at the molecular level, and their influence on the rate and mechanism of drug release. For parenteral nanoemulsion formulation, the encapsulated drug should be released from emulsion droplets after injection to ensure it reached the targeted site of action. ${ }^{44}$ Modified Franz cell method was used to study the release of cefuroxime from the CLN system. In this study, the violation of sink conditions by side-by-side diffusion cell method was overcome by the addition of PBS with the emulsion sample onto the donor compartment by $1: 2$ ratio. The in vitro release profile of CLN is depicted in Figure 6.

Percentage of cumulative release of cefuroxime from both standard solution and CLN exhibited a biphasic pattern, as shown by the rapid initial release of the drug during the first $8 \mathrm{~h}$, followed by a slow release for the next $24 \mathrm{~h}$ (Figure 6). The standard drug solution achieved $81.30 \% \pm 4.22 \%$ of drug release within $8 \mathrm{~h}$, followed by a slower release till it reached a total drug release of $\sim 98.20 \% \pm 3.51 \%$ within $24 \mathrm{~h}$. At this point, the drug had reached the steady-state equilibrium in which no significant increase in the rate of cefuroxime released into the tested medium after $36 \mathrm{~h}$. CLN had a similar release profile as the drug solution but at a slower rate as compared to the standard solution and achieved maximum drug release $87.50 \% \pm 4.22 \%$ after $36 \mathrm{~h}$. CLN presented $>80 \%$ release of drug, the generally acceptable value in the dosage form that should be released from a long-term drug delivery system. ${ }^{45}$ The CLN release curve showed a rapid initial burst (from the small droplets' size) and an extended tail (from larger droplets) that may be attributed to its PDI. ${ }^{44}$ This result was supported by Ritger and Peppas $(1987)^{46}$ who demonstrated the effect of PDI (particle size distribution) on drug release behavior. The sustained release of cefuroxime from nanoemulsion may be attributed to high encapsulation efficiency whereby, in order to be diffused in the aqueous medium, the drug molecules must overcome two different

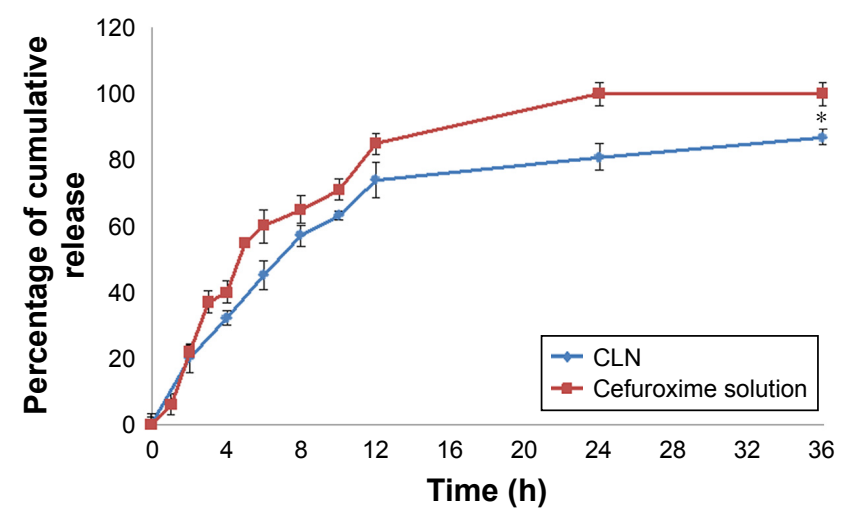

Figure 6 In vitro release of CLN as a function of time compared to cefuroxime standard solution in phosphate buffer, $\mathrm{pH} 7.4$.

Notes: Drug release measurements were carried out in triplicate. Error bars denote standard deviation. *Significant values $(P<0.05)$ in comparison with free cefuroxime solution.

Abbreviation: CLN, cefuroxime-loaded nanoemulsion. 
barriers, such as diffusion across oil core and diffusion through droplet-surfactant interfacial layer. ${ }^{47}$

\section{Encapsulation efficiency study of CLN}

Drug encapsulated within the nanoemulsion system can preciously control the release rate and target drugs to a specific organ. Through the entrapment efficiency (EE) study, the solubility of the drug in a dispersed phase could also be predicted. Thus, the determination of EE has become an integral part of physicochemical properties of the submicron emulsion. The EE of CLN was evaluated using the ultrafiltration method. This method separates the continuous and disperse phases of the emulsion system by allowing the ultrafiltration to take place in the opposite direction of centrifugal force:

$$
\mathrm{EE}(\%)=\frac{\mathrm{WI}-\mathrm{WO}}{\mathrm{WI}} \times 100
$$

where WI is the amount of drug initially present in the formulation and WO is the estimated amount from the aqueous phase of the formulation.

As depicted in Table 3, cefuroxime was found to be completely encapsulated in the oil phase of CLN system. The high percentage obtained indicated the efficiency of the formulation method to entrap the drug into the delivery system. This may be due to the relative lipophilicity of the cefuroxime, as this drug was retained in the oil core of the nanoemulsions. ${ }^{48}$

\section{Stability evaluation of CLN}

In the stability evaluation steps, the nanoemulsions were stored at two different temperatures, which were $25^{\circ} \mathrm{C}$ (room temperature) and $4^{\circ} \mathrm{C}$ for 6 months and monitored with respect to particle size, PDI, and ZP measurements, as previously mentioned in the "Particle size, PDI, and ZP measurements" section.

Stability assessment of CLN at two different temperatures revealed the notable changes in the evaluated parameters, as

Table 3 Encapsulation efficiency data for CLN

\begin{tabular}{ll}
\hline Sample ID & $\begin{array}{l}\text { Encapsulation } \\
\text { efficiency (\%) }\end{array}$ \\
\hline CLNI & $100.45 \pm 2.31$ \\
CLN2 & $100.21 \pm 1.74$ \\
CLN3 & $100.27 \pm 0.93$ \\
\hline
\end{tabular}

Notes: CLNI, CLN2, and CLN3 were samples with different drug loadings of I.0, 2.0 , and $3.0 \mathrm{mg} / \mathrm{mL}$, respectively. All measurements were performed in triplicate. Abbreviation: CLN, cefuroxime-loaded nanoemulsion.
Table 4 Stability evaluation of CLN stored at room temperature $\left(25^{\circ} \mathrm{C}\right)$ in 6 months of the assessment period

\begin{tabular}{llll}
\hline $\begin{array}{l}\text { Storage duration } \\
\text { (months) }\end{array}$ & Size $(\mathbf{n m})$ & PDI & ZP \\
\hline 0 & $100.32 \pm 0.75$ & $0.18 \pm 0.01$ & $-46.90 \pm 1.39$ \\
1 & $99.88 \pm 0.82$ & $0.17 \pm 0.01$ & $-44.40 \pm 0.87$ \\
2 & $103.67 \pm 0.65$ & $0.20 \pm 0.02$ & $-35.90 \pm 2.55$ \\
3 & $109.57 \pm 1.15$ & $0.25 \pm 0.01$ & $-41.30 \pm 1.64$ \\
4 & $107.17 \pm 0.80$ & $0.19 \pm 0.02$ & $-44.60 \pm 1.00$ \\
5 & $121.63 \pm 0.32$ & $0.19 \pm 0.01$ & $-40.50 \pm 1.76$ \\
6 & $141.10 \pm 2.79$ & $0.31 \pm 0.01$ & $-36.80 \pm 0.31$ \\
\hline
\end{tabular}

Note: Measurements have been performed in triplicate.

Abbreviations: $\mathrm{CLN}$, cefuroxime-loaded nanoemulsion; PDI, polydispersity index; ZP, zeta potential.

shown in Tables 4 and 5, respectively. The gradual increase in globule size was observed in CLN sample stored at $25^{\circ} \mathrm{C}$, and the marked change in globule size was noted during the storage period of 5 and 6 months. Even though the PDI and ZP data were within the acceptable limit for parenteral application, a phase separation occurred after a month of storage at this temperature. Since phase separation was a strong indication of physical and chemical nanoemulsion instabilities, CLN was considered not stable at room temperature. Temperature significantly affects the nanoemulsion stability by altering the physical properties of excipients in the oil and aqueous phases of emulsion system including oil, water, interfacial films, and surfactant solubility. ${ }^{49}$ At high temperature, the thermal energy of the droplets increased, and this, in turn, increased the frequency of droplet collisions. Additionally, at high temperature, the interface viscosity was reduced by causing the breakage of the interfacial layer and faster droplet coalescence (fusion of two or more droplets into one larger drop). ${ }^{50}$

From the stability assessment, it was found that CLN exhibited good physical and chemical stabilities when

Table 5 Stability evaluation of CLN stored in humidified condition $\left(4^{\circ} \mathrm{C}\right)$ in 6 months of the assessment period

\begin{tabular}{llll}
\hline $\begin{array}{l}\text { Storage duration } \\
\text { (months) }\end{array}$ & Size $(\mathbf{n m})$ & PDI & ZP \\
\hline 0 & $100.32 \pm 0.75$ & $0.18 \pm 0.01$ & $-46.90 \pm 1.39$ \\
1 & $99.63 \pm 0.15$ & $0.24 \pm 0.01$ & $-41.30 \pm 1.86$ \\
2 & $100.33 \pm 0.15$ & $0.18 \pm 0.01$ & $-38.60 \pm 0.61$ \\
3 & $100.06 \pm 1.54$ & $0.17 \pm 0.01$ & $-36.90 \pm 1.45$ \\
4 & $101.00 \pm 0.43$ & $0.23 \pm 0.01$ & $-33.40 \pm 0.56$ \\
5 & $102.43 \pm 2.06$ & $0.20 \pm 0.01$ & $-30.90 \pm 1.67$ \\
6 & $103.23 \pm 1.25$ & $0.25 \pm 0.01$ & $-42.00 \pm 1.68$ \\
\hline
\end{tabular}

Note: Measurements have been performed in triplicate.

Abbreviations: CLN, cefuroxime-loaded nanoemulsion; PDI, polydispersity index; ZP, zeta potential. 


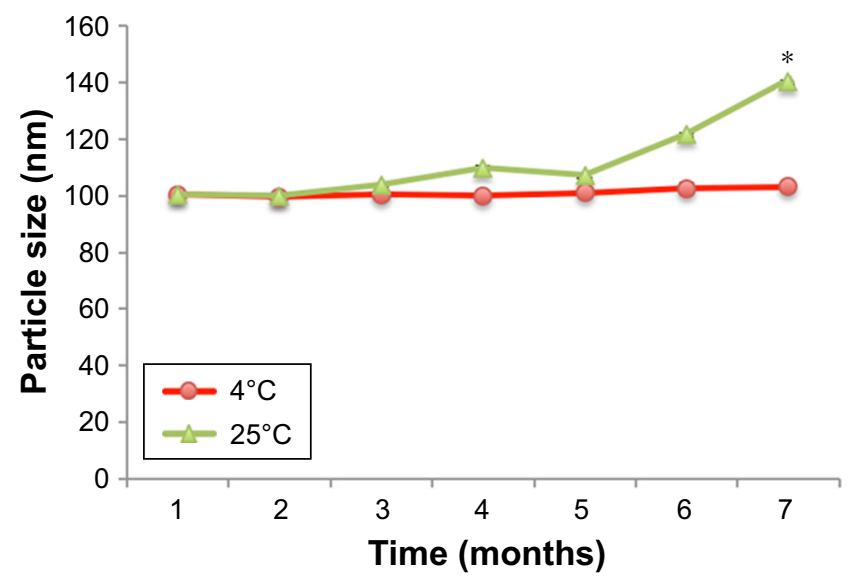

Figure 7 Droplet size of cefuroxime-loaded nanoemulsion under different storage conditions during 6 month stability test.

Notes: All measurements were performed in triplicate. *Significant value $(P<0.05)$ in comparison with the temperature of $4^{\circ} \mathrm{C}$.

stored at $4^{\circ} \mathrm{C}$ (Figure 7 ). The CLN particle size remained largely unchanged during the 6 months of assessment period with only a slight increase at months 4-6. The PDI and ZP values were within the acceptable limit of parenteral application, and no apparent phase separation was observed during the assessment period. The stable particle size of CLN throughout the evaluation period reflected the capability of the CLN system against Ostwald ripening that was characterized by the change in droplet size with the passage of time. Ostwald ripening occurs as a result of molecular diffusion in which droplets from the dispersed phase migrate to the continuous phase. ${ }^{51}$ The excellent stability of CLN may be attributed by the use of the nonionic emulsifier (lecithin) that formed a bulk steric barrier against particle collision. ${ }^{52}$

As presented in Table 6, 2.96\% of drug loss was observed at initial storage, and subsequent drug degradation was observed upon a storage period of 3 and 6 months with 5.59 and $7.57 \%$ of drug loss, respectively. The drug degradation was the challenging aspect of this study since it may affect the chemical and physical stabilities of the emulsion system.

Table 6 Total drug content of CLN with storage at $4^{\circ} \mathrm{C}$ over the 6 month assessment period

\begin{tabular}{llll}
\hline $\begin{array}{l}\text { Time } \\
\text { (months) }\end{array}$ & $\begin{array}{l}\text { Drug loading } \\
\mathbf{( m g / m L )}\end{array}$ & $\begin{array}{l}\text { Drug recovery } \\
\mathbf{( m g / m L )}\end{array}$ & $\begin{array}{l}\text { Drug loss } \\
\mathbf{( \% )}\end{array}$ \\
\hline 0 & $3.04 \pm 5.32$ & $2.95 \pm 4.37^{*}$ & 2.96 \\
3 & $3.04 \pm 5.32$ & $2.87 \pm 6.10^{*}$ & 5.59 \\
6 & $3.04 \pm 5.32$ & $2.81 \pm 3.55^{*}$ & 7.57 \\
\hline
\end{tabular}

Notes: Each value represents the mean \pm standard deviation $(n=3)$. *Significant values relative to the initial drug loading at 0 months (Student's $t$-test, $P<0.05$ ).

Abbreviation: CLN, cefuroxime-loaded nanoemulsion.
However, the drug degradation was a common problem in drugs with functional groups such as esters, amines, and lactam due to hydrolytic degradation. ${ }^{53}$ Cefuroxime belongs to $\beta$-lactam class; thus, some drug loss was expected during the drug content assessment. Another factor that attributed to drug degradation was the auto-oxidation of the oil component in CLN system, but this process was slowed down by storage in humidified condition $\left(4^{\circ} \mathrm{C}\right)$.

\section{Pharmacokinetic profile of CLN Plasma concentration profile}

Detection of cefuroxime in the blood and brain is necessary to evaluate its pharmacological role or clinical application. ${ }^{54}$ The plasma-time concentration profile of cefuroxime after intraperitoneal administration of free cefuroxime solution and CLN is presented in Figure 8. Pharmacokinetic parameters of cefuroxime solution and CLN in plasma are presented in Table 7. The plasma concentration of cefuroxime in CLN-treated rats was significantly higher than free cefuroxime solution at all time intervals. The area under the curve (AUC) $)_{0-t}$ of cefuroxime in plasma from CLN-treated rats $(247.70 \pm 5.76 \mu \mathrm{g} \mathrm{h} / \mathrm{mL})$ was 1.92 -fold higher than that of cefuroxime in plasma from free-cefuroxime solution $(129.15 \pm 4.53 \mu \mathrm{g} \mathrm{h} / \mathrm{mL})$. The half-life $\left(T_{1 / 2}\right)$ of cefuroxime in plasma was also significantly prolonged for CLN-treated rats $(1.61 \pm 0.91 \mathrm{~h})$ as compared to free cefuroxime-treated rats $(1.46 \pm 1.08 \mathrm{~h})$. The total plasma clearance (CL) was reduced by 1.67 -fold in CLN-treated rats as compared to free cefuroxime-treated rats $(P<0.05)$. Although not statistically significant, the elimination rate (Kel) from CLN-treated rats $(0.43 \pm 0.010)$ also reduced by 1.12 -fold compared to free cefuroxime-treated rats $(0.48 \pm 0.04)(P>0.05)$. The prolonged half-life and reduced plasma CL of cefuroxime in CLN indicated that the systemic concentration of cefuroxime was higher in CLN-treated rats than in free cefuroxime-treated rats. Thus, it was suggested that the use of nanoemulsion (CLN) improved cefuroxime permeability across the BBB.

\section{Biodistribution of drug in brain}

The bioavailability of cefuroxime in brain tissues at selected time points was found to be higher in CLN-treated rats than in free cefuroxime-treated rats (Figure 9), in which the brain concentration of cefuroxime was 1.41-fold higher in CLN-treated rats. The pharmacokinetic (PK) data (Table 8) also showed a prolonged half-life and reduced brain CL of cefuroxime, indicating that the encapsulated cefuroxime in nanoemulsion improved the bioavailability of cefuroxime in the brain. 


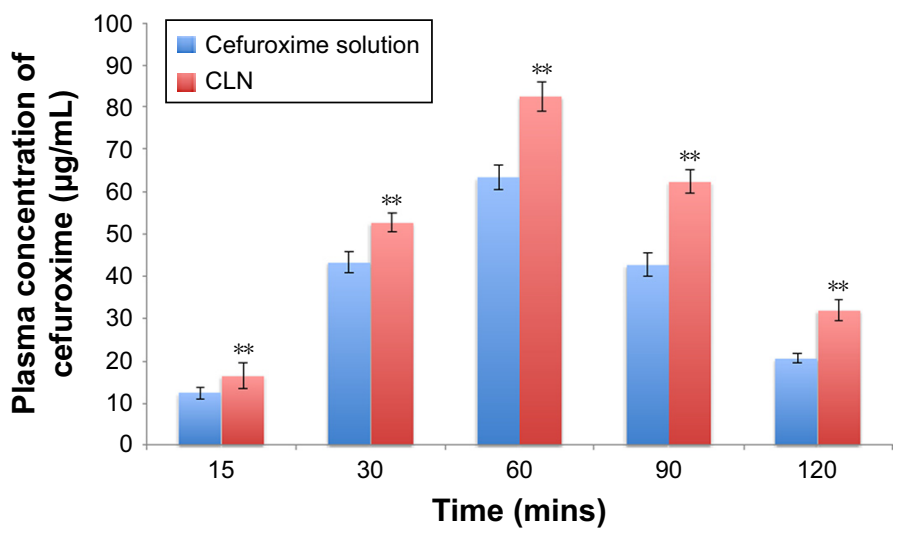

Figure 8 Plasma concentration against time profile after intraperitoneal administration of cefuroxime solution and CLN at the fixed dose of $20 \mathrm{mg} / \mathrm{kg}$ in rats. Notes: Each value represents the mean \pm standard deviation $(n=4)$. **Significant values $(P<0.05)$ in comparison with cefuroxime solution.

Abbreviation: CLN, cefuroxime-loaded nanoemulsion.

From the calculated $K_{\mathrm{p}}$ value, it was found that CLN had a slightly lower value of $K_{\mathrm{p}}$ (Table 9) compared to free cefuroxime solution after the rats were intraperitoneally treated. As the $K_{\mathrm{p}}$ value indicated the extent of brain permeation, this result showed that the brain uptake for both compounds occurred at similar rates despite the high concentration of cefuroxime in CLN-treated plasma. This result may be explained by the use of Tween 80 in CLN formulation that involved receptor-mediated endocytosis of drug molecules across BBB. ${ }^{55}$ This process was not a concentration-dependent process; hence, a higher drug concentration in plasma would not affect the rate of brain uptake. Additionally, Tween 80 enhanced the absorption of plasma protein (apolipoprotein $\mathrm{E}$ ) on the surface of nanocarrier and subsequently increased the brain uptake of Tween-coated nanoemulsion into the brain. ${ }^{56}$

Relatively, the $K_{\mathrm{p}}$ value of $<1$ may result from more extensive binding to plasma proteins than to proteins in the brain tissue and reflected a significant impairment in CNS distribution. ${ }^{57}$ Hence, even though $K_{\mathrm{p}}$ was widely used to

Table 7 Pharmacokinetic parameters of cefuroxime solution and CLN in plasma

\begin{tabular}{lll}
\hline Parameter & Cefuroxime standard & CLN \\
\hline $\mathrm{AUC}_{0-x}(\mu \mathrm{g} \mathrm{h} / \mathrm{mL})$ & $129.15 \pm 4.53$ & $247.70 \pm 5.76^{*}$ \\
$C_{\max }(\mu \mathrm{g} / \mathrm{mL})$ & $63.34 \pm 3.0$ & $82.41 \pm 3.45^{*}$ \\
$T_{\text {max }}(\mathrm{min})$ & 60.00 & 60.00 \\
$T_{1 / 2}(\mathrm{~h})$ & $1.46 \pm 1.08$ & $1.61 \pm 0.9 I^{*}$ \\
$K_{\mathrm{el}}(\mathrm{I} / \mathrm{h})$ & $0.48 \pm 0.04$ & $0.43 \pm 0.0 I^{* *}$ \\
$V_{\mathrm{d}}(\mathrm{L})$ & $0.57 \pm 0.07$ & $0.37 \pm 0.09 *$ \\
$\mathrm{CL}(\mathrm{L} / \mathrm{h})$ & $0.27 \pm 1.32$ & $0.16 \pm 2.1 I^{*}$ \\
\hline
\end{tabular}

Notes: Each value represents the mean \pm standard deviation $(n=4)$. *Significant values $(P<0.05)$ in comparison with cefuroxime solution. ${ }^{*}$ Significant values $(P>0.05)$.

Abbreviations: $\mathrm{CL}$, clearance; $\mathrm{CLN}$, cefuroxime-loaded nanoemulsion. predict the extent of brain permeation, it was argued that the calculated $K_{\mathrm{p}}$ value may be misleading since the total concentration was affected by the nonspecific binding of the molecules to the plasma protein and brain tissue. Additionally, drug transporters and complex interaction within brain fluids have different impacts in drug delivery and CL within CNS compartments. ${ }^{58}$ It was suggested that the unbound brain-to-plasma concentration ratio $K_{\text {pu }}$ was a better parameter that precisely indicated the BBB availability of compounds. ${ }^{59} K_{\mathrm{pu}}$ describes the free drug concentration of the drug molecule in the brain and can be directly measured through micro dialysis probe in the brain. However, this method is experimentally challenging and involves a lot of

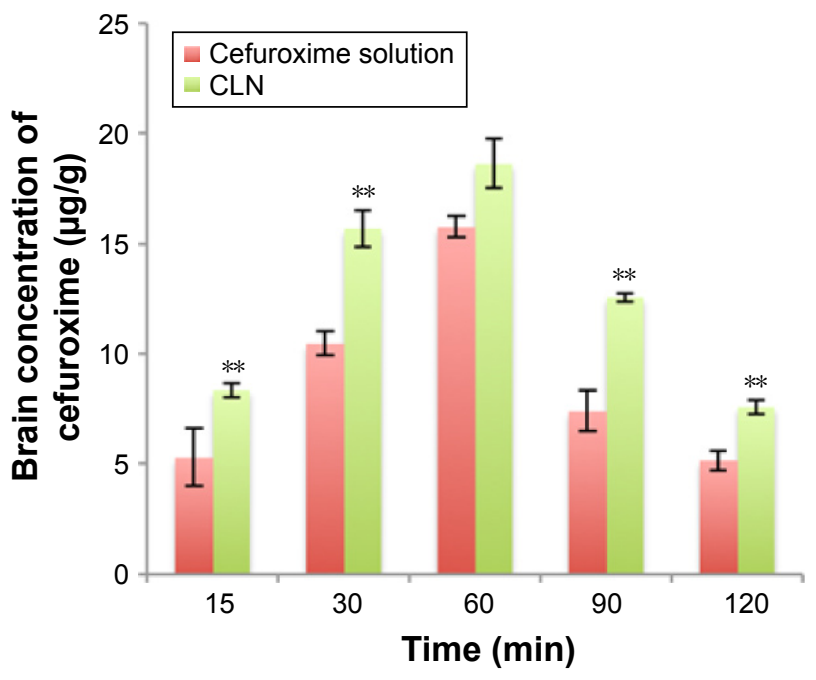

Figure 9 Brain concentration against time profile after intraperitoneal administration of cefuroxime solution and CLN at the fixed dose of $20 \mathrm{mg} / \mathrm{kg}$ in rats.

Notes: Each value represents the mean \pm standard deviation $(n=4)$. **Significant values $(P<0.0 \mathrm{I})$ in comparison with cefuroxime solution.

Abbreviation: CLN, cefuroxime-loaded nanoemulsion. 
Table 8 Pharmacokinetic parameters of cefuroxime solution and CLN in the brain

\begin{tabular}{lll}
\hline Parameter & Cefuroxime standard & CLN \\
\hline $\mathrm{AUC}_{0-x}(\mu \mathrm{g} \mathrm{h} / \mathrm{mL})$ & $31.97 \pm 5.98$ & $45.26 \pm 3.54^{* *}$ \\
$C_{\max }(\mu \mathrm{g} / \mathrm{mL})$ & $15.78 \pm 3.21$ & $18.63 \pm 2.45$ \\
$T_{\max }(\mathrm{min})$ & 60.0 & 60.0 \\
$T_{1 / 2}(\mathrm{~h})$ & $0.93 \pm 0.67$ & $1.97 \pm 0.91$ \\
$K_{\mathrm{el}}(\mathrm{I} / \mathrm{h})$ & $0.74 \pm 0.05$ & $0.35 \pm 0.05^{* *}$ \\
$V_{d}(\mathrm{~L})$ & $0.68 \pm 0.06$ & $0.43 \pm 0.04^{* *}$ \\
$\mathrm{CL}(\mathrm{L} / \mathrm{h})$ & $0.51 \pm 3.22$ & $0.15 \pm 1.79 * *$ \\
\hline
\end{tabular}

Notes: Each value represents the mean \pm standard deviation $(n=4)$. **Significant values $(P<0.05)$ in comparison with cefuroxime solution.

Abbreviations: $\mathrm{CL}$, clearance; CLN, cefuroxime-loaded nanoemulsion.

Table $9 \mathrm{~K}_{\mathrm{p}}$ was calculated as the ratio of brain tissue concentration to plasma level

\begin{tabular}{lll}
\hline & Cefuroxime solution & CLN \\
\hline Brain-to-plasma ratio $\left(K_{\mathrm{p}}\right)$ & $0.71 \pm 0.02$ & $0.69 \pm 0.01 *$ \\
\hline
\end{tabular}

Notes: Each value represents the mean \pm standard deviation $(n=4)$. *Significant values $(P<0.05)$ in comparison with cefuroxime solution.

Abbreviation: CLN, cefuroxime-loaded nanoemulsion.

resources to carry out. In future research, $K_{\mathrm{pu}}$ can be considered to gain a better understanding of the PK profile.

\section{Conclusion}

CLN formulation was feasible for parenteral administration and exhibited an excellent physicochemical stability as demonstrated by particle size, PDI, and ZP measurements within 6 months of assessment period. The viscosity value of cefuroxime was found to be $1.24 \pm 0.34 \mathrm{mPa}$ s and within the specification for parenteral emulsions. Through the entrapment efficiency (EE) study, cefuroxime was found to be completely encapsulated in the oil phase of the nanoemulsion system. For the microscopic study, TEM photomicrograph of CLN was in agreement with the result obtained by the DLS method. The in vitro release profile of CLN followed a sustained-release pattern, and MTT assay revealed that CLN was nontoxic at the concentration of $10 \mu \mathrm{g} / \mathrm{mL}$. The pharmacokinetic profile of CLN showed significantly higher $C_{\text {max }}, \mathrm{AUC}_{0-t}$, prolonged half-life, and lower total plasma CL indicating that the systemic concentration of cefuroxime was higher in CLN-treated rats than in free cefuroxime-treated rats. A similar profile was obtained for the cefuroxime biodistribution in the brain in which CLN showed a significantly higher $C_{\max }, \mathrm{AUC}_{0-t}$, prolonged half-life, and lower CL as compared to the free cefuroxime solution. $K_{\mathrm{p}}$ value of CLN had a significantly equal or slightly lower value of $K_{\mathrm{p}}$ compared to the free cefuroxime solution despite the high concentration of cefuroxime in CLN-treated plasma. CLN also showed improved pharmacokinetic profile in vivo as compared to the free cefuroxime solution that reflected its practical approach to enhance cefuroxime delivery to the brain.

\section{Acknowledgments}

The authors would like to thank the Research University Grant Scheme (RUGS), Universiti Putra Malaysia, for funding this project under the project number UPM/700-1/2/ RUGS/04-02-12-1762RU. The brain microvascular endothelial cells (hCMEC/D3) were kindly donated by the Institute of Cochin, INSERM, Paris, and the use of the cells in this study has received UPM-INSERM review board approval.

\section{Disclosure}

The authors report no conflicts of interest in this work.

\section{References}

1. Swartz MN, Dodge PR. Bacterial meningitis - a review of selected aspects. N Engl J Med. 1965;272(15):779-787.

2. Honda H, Warren DK. Central nervous system infections: meningitis and brain abscess. Infect Dis Clin North Am. 2009;23(3):609-623.

3. Manten A. Side effects of antibiotics. Vet Q. 1981;3(4):179-182.

4. Yılmaz Ç, Özcengiz G. Antibiotics: pharmacokinetics, toxicity, resistance and multidrug efflux pumps. Biochem Pharmacol. 2017; 133:43-62.

5. Pardridge WM. The blood-brain barrier: bottleneck in brain drug development. NeuroRx. 2005;2(1):3-14. doi:10.1602/neurorx.2.1.3.

6. Zivanovic L, Ivanovic I, Vladimirov S, Zecevic M. Investigation of chromatographic conditions for the separation of cefuroxime axetil and its geometric isomer. J Chromatogr B Analyt Technol Biomed Life Sci. 2004;800:175-179.

7. Kossmann T, Hans V, Stocker R, et al. Penetration of cefuroxime into the cerebrospinal fluid of patients with traumatic brain injury. J Antimicrob Chemother. 1996;37:161-167.

8. Nau R, Sörgel F, Eiffert H. Penetration of drugs through the bloodcerebrospinal fluid/blood-brain barrier for treatment of central nervous system infections. Clin Microbiol Rev. 2010;23(4):858-883.

9. Date AA, Nagarsenker MS. Parenteral microemulsions : an overview. Int J Pharm. 2008;355:19-30.

10. Lovelyn C, Attama AA. Current state of nanoemulsions in drug delivery. J Biomater Nanobiotechnol. 2011;2(5):626-639.

11. Weksler BB, Subileau EA, Perrière N, et al. Blood-brain barrier-specific properties of a human adult brain endothelial cell line. FASEB J. 2005;19(13):1872-1874.

12. Bari H. A prolonged release parenteral drug delivery system - an overview. Int J Pharm Sci Rev Res. 2010;3(1):1-11.

13. Prabhakar K, Afzal SM, Surender G, Kishan V. Tween 80 containing lipid nanoemulsions for delivery of indinavir to brain. Acta Pharm Sin B. 2013;3(5):345-353.

14. Araújo FA, Kelmann RG, Araújo BV, Finatto RB, Teixeira HF, Koester LS. European Journal of Pharmaceutical Sciences Development and characterization of parenteral nanoemulsions containing thalidomide. Eur J Pharm Sci. 2011;42:238-245.

15. Avellini L, Terracina L, Gaiti A. Linoleic acid passage through the blood-brain barrier and a possible effect of age. Neurochem Res. 1994; 19(2):129-133.

16. Mitchell RW, On NH, Del Bigio MR, Miller DW, Hatch GM. Fatty acid transport protein expression in human brain and potential role in fatty acid transport across human brain microvessel endothelial cells. J Neurochem. 2011;117(4):735-746. 
17. Hsu JP, Nacu A. Behavior of soybean oil-in-water emulsion stabilized by nonionic surfactant. J Colloid Interface Sci. 2003;259(2):374-381.

18. Lu Y, Zhang Y, Yang Z, Tang X. Formulation of an intravenous emulsion loaded with a clarithromycin-phospholipid complex and its pharmacokinetics in rats. Int J Pharm. 2009;366(1-2):160-169.

19. Skouroliakou M, Kountouri AM, Hatziantoniou S, Koutri K, Chiou A Physicochemical stability assessment of all-in-one parenteral emulsion for neonates containing SMOFlipid. BMJ. 2012;19:514-518.

20. van Hoogevest P, Wendel A. The use of natural and synthetic phospholipids as pharmaceutical excipients. Eur J Lipid Sci Technol. 2014; 116(9):1088-1107.

21. FDA [webpage on the Internet]. Inactive Ingredient Search for Approved Drug Products; 2010. Available from: https://www.accessdata.fda.gov/ scripts/cder/iig/getiigWEB.cfm. Accessed August 23, 2017.

22. Koffie RM, Farrar CT, Saidi L-J, William CM, Hyman BT, SpiresJones TL. Nanoparticles enhance brain delivery of blood-brain barrierimpermeable probes for in vivo optical and magnetic resonance imaging. Proc Natl Acad Sci U S A. 2011;108(46):18837-18842.

23. Weiss CK, Kohnle M, Landfester K, Hauk T. The first step into the brain: uptake of NIO-PBCA nanoparticles by endothelial cells in vitro and in vivo, and direct evidence for their blood-brain barrier permeation. ChemMedChem. 2008;3:1395-1403.

24. Kachel DL, Moyer TP, Martin WJ. Amiodarone-induced injury of human pulmonary artery endothelial cells: protection by alphatocopherol. J Pharmacol Exp Ther. 1990;254(3):1107-1112.

25. Goulet $\mathrm{O}$, Antebi $\mathrm{H}$, Wolf C, et al. Original communication. J Parenter Enter Nutr. 2010;34(5):485-495.

26. Zhang HY, Tang X, Li HY, Liu XL. A lipid microsphere vehicle for vinorelbine: stability, safety and pharmacokinetics. Int $J$ Pharm. 2008;348(1-2):70-79.

27. Bernardi DS, Pereira TA, Maciel NR, et al. Formation and stability of oil-in-water nanoemulsions containing rice bran oil: in vitro and in vivo assessments. J Nanobiotechnology. 2011;9:44.

28. Anuchapreeda S, Fukumori Y, Okonogi S, Ichikawa H. Preparation of lipid nanoemulsions incorporating curcumin for cancer therapy. Journal of Nanotechnology. 2012;2012:11.

29. Markoutsa E, Papadia K, Clemente C, Flores O, Antimisiaris SG. Anti-AB-MAb and dually decorated nanoliposomes: effect of AB1-42 peptides on interaction with hCMEC/D3 cells. Eur J Pharm Biopharm. 2012;81(1):49-56.

30. Peng J, Dong WJ, Li L, et al. Effect of high-pressure homogenization preparation on mean globule size and large-diameter tail of oil-in-water injectable emulsions. J Food Drug Anal. 2015;23(4):828-835.

31. Wang JJ, Sung KC, Hu OYP, Yeh CH, Fang JY. Submicron lipid emulsion as a drug delivery system for nalbuphine and its prodrugs. $J$ Control Release. 2006;115(2):140-149.

32. Jo YJ, Kwon YJ. Characterization of $\beta$-carotene nanoemulsions prepared by microfluidization technique. Food Sci Biotechnol. 2014;23(1) 107-113.

33. Larsson M, Hill A, Duffy J. Suspension stability; why particle size, zeta potential and rheology are important. Annu Trans Nord Rheol Soc. 2012;20:209-214.

34. Elsheikh MA, Elnaggar YS, Gohar EY, Abdallah OY. Nanoemulsion liquid preconcentrates for raloxifene hydrochloride: optimization and in vivo appraisal. Int J Nanomedicine. 2012;7:3787.

35. Levy MY, Benita S. Design and characterization of a submicronized o/w emulsion of diazepam for parenteral use. Int J Pharm. 1989;54(2): 103-112.

36. Pichot R, Watson R, Norton I. Phospholipids at the interface: current trends and challenges. Int J Mol Sci. 2013;14(6):11767-11794.

37. Patel R. Parenteral suspension: an overview. Int J Curr Pharm Res. 2010; 2(3):4-13.

38. Roethlisberger D, Mahler H-C, Altenburger U, Pappenberger A If euhydric and isotonic do not work, what are acceptable ph and osmolality for parenteral drug dosage forms? J Pharm Sci. 2017;106(2): 446-456.
39. Schuh RS, Bruxel F, Teixeira HF. Physicochemical properties of lecithin-based nanoemulsions obtained by spontaneous emulsification or high-pressure homogenization. Quim Nova. 2014;37(7):1193-1198.

40. Mattos C, Argenta D, Melchiades G, et al. Nanoemulsions containing a synthetic chalcone as an alternative for treating cutaneous leshmaniasis: optimization using a full factorial design. Int J Nanomedicine. 2015; $10: 5529$

41. Niles AL, Moravec RA, Riss TL. In vitro viability and cytotoxicity testing and same-well multi-parametric combinations for high throughput screening. Curr Chem Genomics. 2009;3:33-41.

42. Azeem A, Rizwan M, Ahmad FJ, et al. Brief/technical note nanoemulsion components screening and selection: a technical note. AAPS PharmSciTech. 2009;10(1):69-76.

43. Warisnoicharoen W, Lansley AB, Lawrence MJ. Toxicological evaluation of mixtures of nonionic surfactants, alone and in combination with oil. J Pharm Sci. 2003;92(4):859-868.

44. Salmela L, Washington C. A continuous flow method for estimation of drug release rates from emulsion formulations. Int J Pharm. 2014; 472(1-2):276-281.

45. Kastellorizios M, Burgess DJ. In vitro drug release testing and in vivo/in vitro correlation for long acting implants and injections. Long Acting Injections and Implants. Boston, MA: Springer US; 2012: 475-503.

46. Ritger PL, Peppas NA. A simple equation for description of solute release II. Fickian and anomalous release from swellable devices. J Control Release. 1987;5(1):37-42.

47. Buyukozturk F, Benneyan JC, Carrier RL. Impact of emulsion-based drug delivery systems on intestinal permeability and drug release kinetics. J Control Release. 2010;142(1):22-30.

48. Ganta S, Amiji M. Coadministration of paclitaxel and curcumin in nanoemulsion formulations to overcome multidrug resistance in tumor cells. Mol Pharm. 2009;6(3):503-511.

49. Li P, Lu W. Food hydrocolloids effects of storage conditions on the physical stability of D-limonene nanoemulsion. Food Hydrocolloids. 2016;53:218-224.

50. Jones T, Neustadter KP, Whittingham K. Water-in-crude oil emulsion stability and emulsion destabilization by chemical this article begins on the next page. J Can Pet. 1978;2(8):100-108.

51. Setya S, Talegaonkar S, Razdan BK. ISSN $2278-4357$ nanoemulsions: formulation methods and stability aspects. World Journal of Pharmacy and Pharmaceutical Sciences. 2014;3(2):2214-2228.

52. Zainol S, Basri M, Basri H, et al. Formulation optimization of a palm-based nanoemulsion system containing levodopa. Int J Mol Sci. 2012;13(10):13049-13064.

53. Deshpande AD, Baheti KG, Chatterjee NR. Degradation of B-lactam antibiotics. Curr Sci. 2004;87(12):1684-1695.

54. Tsai TH, Cheng FC, Chen KC, Chen YF, Chen CF. Simultaneous measurement of cefuroxime in rat blood and brain by microdialysis and microbore liquid chromatography. Application to pharmacokinetics. J Chromatogr B Biomed Sci Appl. 1999;735(1):25-31.

55. Wagner S, Zensi A, Wien SL, et al. Uptake mechanism of ApoE-modified nanoparticles on brain capillary endothelial cells as a blood-brain barrier model. PLoS One. 2012;7(3):3-9.

56. Alexis F, Pridgen E, Molnar LK, Farokhzad OC. Factors affecting the clearance and biodistribution of polymeric nanoparticles. Mol Pharm. 2008;5(4):505-515.

57. Kalvass JC, Maurer TS, Pollack GM. Use of plasma and brain unbound fractions to assess the extent of brain distribution of 34 drugs: comparison of unbound concentration ratios to in vivo P-glycoprotein efflux ratios. Drug Metab Dispos. 2007;35(4):660-666.

58. Jeffrey P, Summerfield S. Assessment of the blood brain bariier in CNS drug discovery. Neurobiol Dis. 2010;37:33-37. doi:10.1016/j.nbd. 2009.07.033

59. Reichel A. Addressing central nervous system (CNS) penetration in drug discovery: basics and implications of the evolving new concept. Chem Biodivers. 2009;6(11):2030-2049. 


\section{Publish your work in this journal}

The International Journal of Nanomedicine is an international, peerreviewed journal focusing on the application of nanotechnology in diagnostics, therapeutics, and drug delivery systems throughout the biomedical field. This journal is indexed on PubMed Central, MedLine, CAS, SciSearch $\AA$, Current Contents $\AA /$ Clinical Medicine,

Journal Citation Reports/Science Edition, EMBase, Scopus and the Elsevier Bibliographic databases. The manuscript management system is completely online and includes a very quick and fair peer-review system, which is all easy to use. Visit http://www.dovepress.com/ testimonials.php to read real quotes from published authors.

Submit your manuscript here: http://www.dovepress.com/international-journal-of-nanomedicine-journal 